\title{
Modelling for ABS Bench Detection Method
}

\author{
Ruru Hao*, Zhou Zhou and Lan Yang \\ School of Information Engineering, Chang'an University, Middle Section of Nan Er Huan Road, Xi'an 710064, P. R. China \\ ${ }^{*}$ Corresponding author
}

\begin{abstract}
For the high cost, large land occupation and low detection efficiency of auto ABS road experiment, an auto ABS indoor bench detection principle was proposed to make the detection of the integrative performance of auto ABS conveniently. The bench detection system mainly consists of road adhesion coefficient simulation unit, auto motion inertia simulation unit, measurement \& control system and data acquisition system. In order to verify the correctness of the bench detection principle, the vehicle model, wheel model, braking force model, and tire-road model on the bench are established according to the ABS bench detection scheme. The simulation results show that the characteristic of the simulation curves are consistent with the ABS braking curves, which indicates the feasibility of the bench detection principle.
\end{abstract}

Keywords- auto; anti-lock braking system; bench detection; modeling and simulation

\section{INTRODUCTION}

Automobile Anti-lock Braking System (ABS) is one of the most important active safety devices. It can prevent the wheel from locking up by regulating the slip of the wheel at its optimum value. This permits the driver to control the direction of the vehicle by steering input and achieve the shortest braking distance during emergency braking or braking on slippery roads ${ }^{[1-2]}$. So it can help improve the safety performance of the vehicle by regularly ABS detection.

At present, there are several ABS detection methods including static testing before assembling, Hardware-in-theLoop (HIL) simulation, road experiment, etc. The ABS static testing can check the electrical part and the off/on action of solenoid valve. However, it cannot ensure the matching performance after ABS assembly ${ }^{[3]}$. HIL combines the actual ABS controller with a numerical simulation environment to form a closed-loop test system to test the response of ABS. But, this approach is mainly used into type-match experiment for the specified vehicle. It is not suitable for the periodic testing of the in-use vehicles since the complex vehicle dynamics model and various pavement mathematical models are required. The road experiment performs real vehicle braking on a dedicated road, which can objectively reflect the ABS performance. But the disadvantages of large area occupation, high cost of the test site, susceptible to environment influences and poor reproducibility make it difficult to implement ${ }^{[4]}$. Known for the advantages of safety, high efficiency, small area occupation and invulnerable to environment influences, ABS bench detection has gained much research interest recently. In this paper, an auto ABS indoor bench detection principle was proposed and the simulation model was established to verify the correctness of the proposed method.

\section{Bench-BASED Abs Detection Scheme}

The planned structure of the ABS detection bench is shown in Figure I. It mainly consists of rolling drum, torque controller, flywheel, pressure sensor, speed sensor, motor, etc. The rolling drum, likes the constantly moving road, is used to support the wheel. The torque controller is applied to generate the desired "ground braking force", which varies with the adhesion coefficient. So different adhesion coefficients can be dynamically simulated through change the torques produced by the torque controllers. Translational inertia of vehicle braking on road can be simulated by the rotational inertia of the flywheels on the bench. So the motion of the flywheels on the bench can stand for the motion of vehicle on the road. Since the drum has the same motion with the wheel in the detection process, the wheel motion can be represented by the drum. Wheel speed and vehicle body speed can be respectively measured by sensors installed on drum and that coaxial with the flywheels ${ }^{[5]}$.

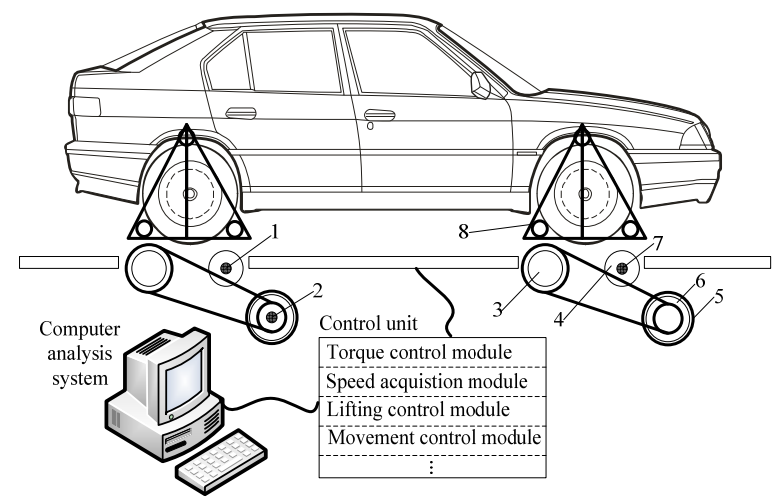

FIGURE I. PLANNED STRUCTURE OF ABS DETECTION BENCH. 1 AND 7 ARE WHEEL SPEED SENSORS, 2 IS VEHICLE SPEED SENSOR, 3 AND 4 ARE DRUMS, 5 IS FLY WHEELS, 6 IS TORQUE CONTROLLER AND 8 IS SECURITY DEVICE.

\section{MODELING AND SimULATION}

The converted drum speed and the flywheel speed are respectively used to represent the wheel speed and the vehicle speed. That means the motion models of the drum and flywheel will separately stand for those of wheel and vehicle. The model of "ground braking force", acting on the flywheel and the drum, will be built according to the torque produced by the torque controller. Tire-road model will generate different adhesion coefficients, which will affect the "ground braking force" on the bench. 


\section{A. Vehicle and Wheel Model on the Bench}

The simulation model of vehicle and wheel on the detection bench can be created according to the forces of the wheel on the bench, shown as Figure II. Where $M_{\mathrm{b}}$ is the braking torque from the brake, $M_{c}$ is the output torque of the torque controller.

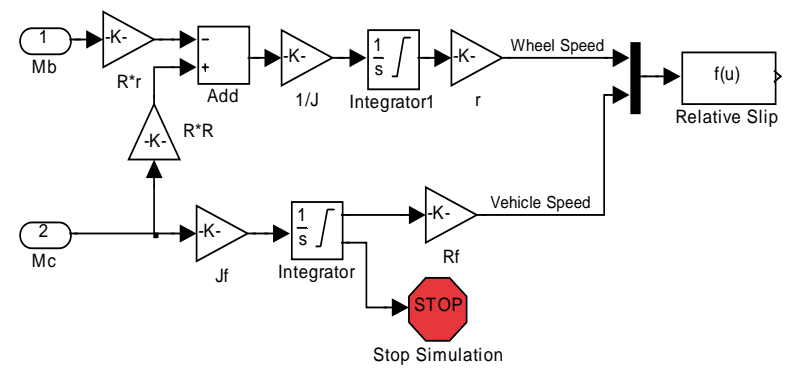

FIGURE II. VEHICLE AND WHEEL SIMULATION MODEL ON THE $\mathrm{BENCH}$

\section{B. Braking Force Model on the Bench}

Braking force on the bench, which is produced by the torque controller, is equivalent to the ground braking force on the road. For a given vehicle, the braking force on the bench is proportional to the adhesion coefficient. Therefore, the desired adhesion coefficient can be simulated by changing the output torque of torque controller. So the Matlab/Simulink simulation model of the braking force on the bench can be established according to the relationship between the desired $\mu$ and the output torque of the torque controller, shown in Figure III.

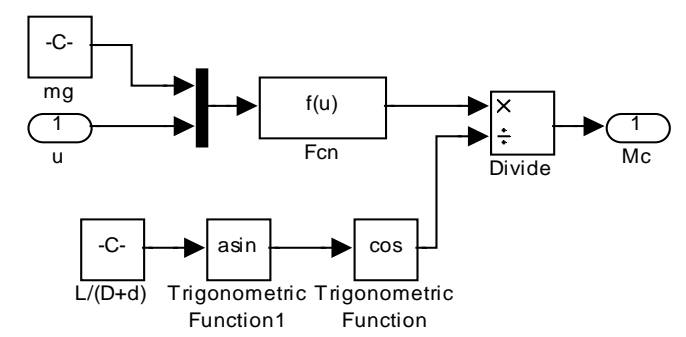

FIGURE III. BRAKING FORCE MODEL ON THE BENCH

\section{Tire-Road Model}

Since the tire and the drum keep relatively static when braking, the tire-road model in the bench detection system is reflected in the constraint of the braking force. That means make the braking force changes are consistent with the tireroad model law on the road. Magic Formula uses a combination of trigonometric formulas to express the steadystate conditions of the tire characteristic parameters. It has a higher fitting accuracy both for the longitudinal force, lateral force or aligning torque. So it is suitable for automobile dynamic simulation and experimental comparison, etc. And it also has been the most widely used model for vehicle dynamics simulation. Therefore, the Magic Formula is used as the tireroad model in this paper. The expression of the Magic Formula is shown in equation (1).

$$
\begin{aligned}
\mu_{L} & =\mu\left(S, B_{L}, C_{L}, D_{L}, E_{L}\right) \\
& =D_{L} \sin \left\{C_{L} \arctan \left[B_{L} S-E_{L}\left(B_{L} S-\arctan \left(B_{L} S\right)\right)\right]\right\}
\end{aligned}
$$

Where $\mu_{L}$ is the longitudinal adhesion coefficient, $B_{L}$ is the stiffness factor, $C_{L}$ is the shape factor, which determines the shape of the curve, $D_{L}$ is the crest factor, which means the maximum value of the curve, $E_{L}$ is the curvature factor, which determines the shape of the curve near the maximum value ${ }^{[6]}$.

According to the equation (1), the Matlab/Simulnk simulation model of tire-road can be established as Figure IV.

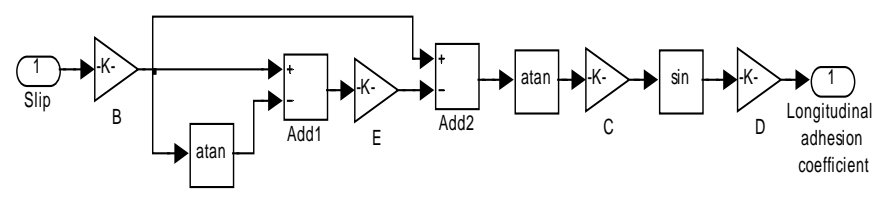

FIGURE IV. TIRE-ROAD MODEL

\section{Single Wheel Simulation Model}

The Matlab/Simulink simulation model of a single wheel vehicle braking on the bench is built through combining the sub-models described above, shown in Figure V. The "Subsys_ABSControl" and "Subsys_Brake" adopt the typical anti-lock braking system and hydraulic system simulation model. "Subsys_uControl" is the tire-road model that to restrict the braking force on the bench. And "Subsys_Mc" is the braking force that produced by torque controller. After running this model, the speed curves of vehicle and wheel can be seen in the scope "Speed curve", the brake distance curve is shown in the scope "Brake distance curve" and slip rate curve can be got in the scope "Slip".

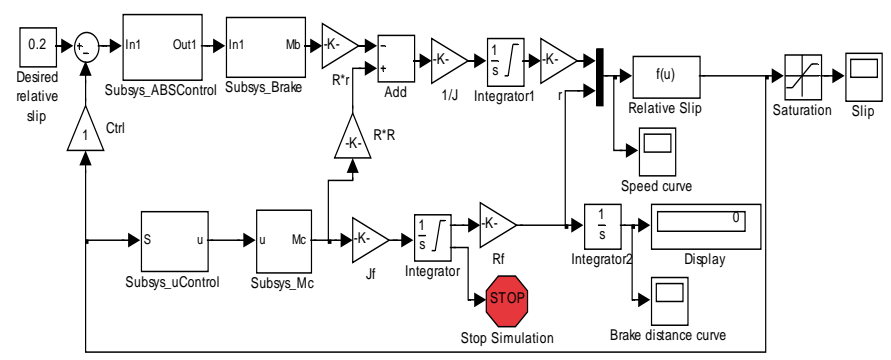

FIGURE V. SINGLE WHEEL SIMULATION MODEL

Set the peak value of the high adhesion coefficient as 0.75 , the peak value of the low adhesion coefficient as 0.15 , and the initial braking speed as $25 \mathrm{~m} / \mathrm{s}$. Figure 6 is the high adhesion coefficient brake simulation curves on bench and Figure 7 is the low adhesion coefficient brake simulation curves on bench. It can be seen from Figure VI and Figure VII that the change trend of the speeds curve and slip rate curves are consistent with the characteristic of the ABS braking curves, which illustrates that the bench detection system can successfully accomplish the ABS detection. 


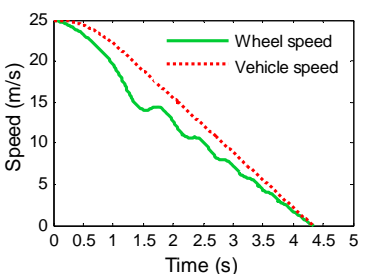

(A) SPEED

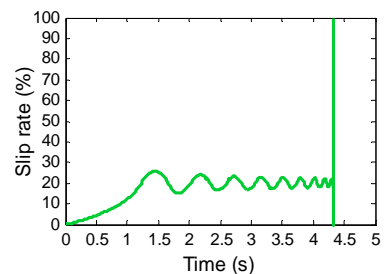

(B) SLIP RATE
FIGURE VI. HIGH ADHESION COEFFICIENT SIMULATION ON BENCH

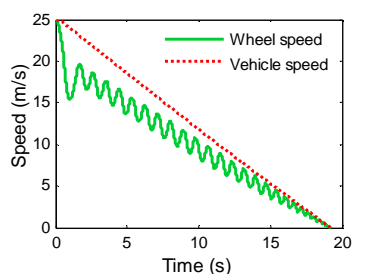

(A) SPEED

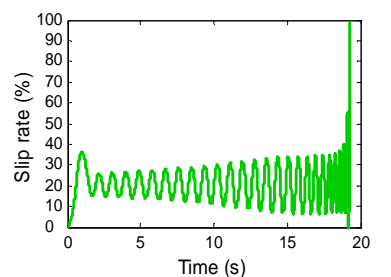

(B) SLIP RATE

FIGURE VII. LOW ADHESION COEFFICIENT SIMULATION ON BENCH

\section{CONCLUSIONS}

In this paper, the research on automobile ABS bench detection principle and model had been carried out. The main work includes three parts. Firstly, the bench-based ABS detection scheme was proposed according to the ABS road experiment theory. The scheme mainly specified the implementation approaches for various road conditions, different adhesion coefficients, and translational inertia of the vehicle and so on. Secondly, the vehicle and wheel model, braking force model, tire-road model and the single wheel simulation model for the ABS bench detection were established. Finally, high and low adhesion coefficient simulation experiments were conducted. And the simulation results indicated a good consistent with the characteristic of the ABS braking, which verified the correctness and feasibility of the proposed bench detection scheme.

\section{ACKNOWLEDGMENT}

This work was partially supported by the National Natural Science Foundation of China (No. 51278058), Program for Changjiang Scholars and Innovative Research Team in University of Ministry of Education of China (No. IRT0951) and the Special Fund for Basic Scientific Research of Central Colleges, Chang'an University in China (310824164004, 310824164006, 310824164007).

\section{REFERENCES}

[1] P. L. William, L. Antonio and G. Mathieu, "Design and experimental validation of a nonlinear wheel slip control algorithm”, Automatica, vol.48, pp.1852-1859, 2012.

[2] D. Savitski, V. Ivanov, K. Augsburg, et al. The new paradigm of an antilock braking system for a full electric vehicle: experimental investigation and benchmarking. Proceedings of the institution of mechanical engineers part D-Journal of automobile engineering, vol.230(10), pp: 1364-1377, 2016.
[3] M. Mirzaei and H. Mirzaeinejad, "Optimal design of a non-linear controller for anti-lock braking system”, Transportation Research Part C, vol.24, pp.19-35, 2012.

[4] X. Q. Zhang, B. Yang, et al, "Research on ABS of multi-axle truck based on ADAMS/Car and Matlab/Simulink”, Procedia Engineering, vol.37, pp. 120-124, 2012.

[5] L. Hartikainen, F. Petry, Westermann Stephan, Longitudinal wheel slip during ABS braking, Vehicle system dynamics, vol.53(2), pp.237-255, 2015.

[6] D. Z. Huang and J. B. Shen, "Study on mathematical model of test bench for vehicle anti-lock braking system”, Proc. of the 2th International Conf. on Intelligent Computation Technology and Automation, Changsha, Hunan, vol.2, pp.268-270, 2009. 